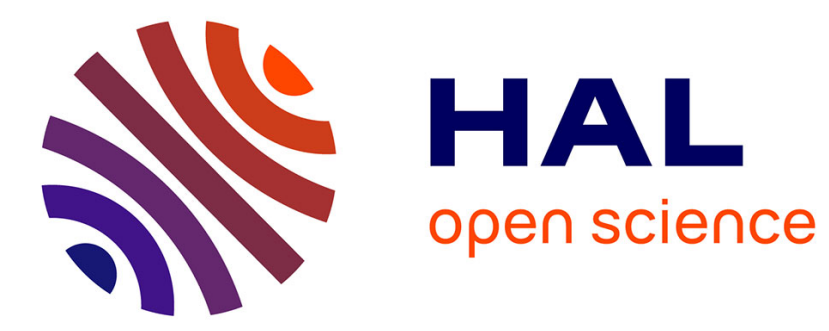

\title{
CRITICAL PHENOMENA AND THE METAL-NONMETAL TRANSITION IN LIQUID METALS
}

F. Hensel, M. Stolz, G. Hohl, R. Winter, W. Götzlaff

\section{- To cite this version:}

F. Hensel, M. Stolz, G. Hohl, R. Winter, W. Götzlaff. CRITICAL PHENOMENA AND THE METALNONMETAL TRANSITION IN LIQUID METALS. Journal de Physique IV Proceedings, 1991, 01 (C5), pp.C5-191-C5-205. 10.1051/jp4:1991523 . jpa-00250647

\section{HAL Id: jpa-00250647 https://hal.science/jpa-00250647}

Submitted on 1 Jan 1991

HAL is a multi-disciplinary open access archive for the deposit and dissemination of scientific research documents, whether they are published or not. The documents may come from teaching and research institutions in France or abroad, or from public or private research centers.
L'archive ouverte pluridisciplinaire HAL, est destinée au dépôt et à la diffusion de documents scientifiques de niveau recherche, publiés ou non, émanant des établissements d'enseignement et de recherche français ou étrangers, des laboratoires publics ou privés. 


\title{
CRITICAL PHENOMENA AND THE METAL-NONMETAL TRANSITION IN LIQUID METALS
}

\author{
F. HENSEL, M. STOLZ, G. HOHL, R. WINTER and W. GÖTZLAFF \\ Institute of Physical Chemistry and Materials Science Center, Philipps University of Marburg, D-3550 \\ Marburg, Germany
}

\begin{abstract}
Experimental results for fluid metals near the liquid-vapour critical point show that profound changes in the electronic structure of fluid metals occur in that region. A gradual transition from metallic to non-metallic behaviour occurs with decreasing density, which manifests itself in a corresponding strong thermodynamic state dependence of the interparticle interaction. The existence of this transition noticeably influences the thermodynamic features of the vapour-liquid phase transition of metals. Another equally important aspect of the problem is the size-dependent gradual evolution of metallic properties in isolated, microscopic metal clusters which clearly must be part of any detailed consideration of the liquid-vapour critical point phase transition of metals.
\end{abstract}

\section{Introduction}

An important open problem in the general field of liquid-vapour critical phenomena is the understanding of the critical point phase transition in metallic fluids. One of the fundamental conceptual difficulties in dealing with such fluids is that the electronic structure of the two coexisting phases, liquid and vapour, may be fundamentally different. Far below the critical point temperature the vapour phase is nonmetallic and well characterized by highly polarizable atoms which interact through weak van der Waals forces, whereas valence electrons in the coexisting metallic liquid phase are dissociated and the interparticle interactions are thought to arise from screened Coulomb potentials. Both of these interactions can be described by short-range potentials /1/ resembling those of molecular fluids so that critical phenomena typical of nonmetallic molecular fluids can be expected. However, both types of interactions change with the thermodynamic state. Nearer to the critical point, the liquid density is much less and the vapour density much greater. At what density does the vapour become metallic, or the liquid nonmetallic? It is this variation of the interaction in the neighbourhood of the metal-nonmetal transition that is difficult to deal with theoretically and which may have a strong influence on the liquid-vapour critical point phase transition. 
The most significant experiments relevant to the effect of the liquid-vapour interparticle asymmetry and to the interplay between the metal-nonmetal transition and the critical point density fluctuations are those on the equation of state and the electronic properties in the immediate neighbourhood of the critical points of metals. However, such data are difficult to study for most metals because of the high temperatures and pressures involved. This is immediately evident from a glance at Table 1 which lists the critical data for those metals whose critical points lie within the limits of static temperatures available in the laboratory.

Table 1 Critical temperatures, pressures and densities of some metals

$\begin{array}{lllll}\text { Metal } & \mathrm{T} / \mathrm{K} & \mathrm{p} / \mathrm{bar} & \rho / \mathrm{gcm}^{-3} & \text { Ref. } \\ \mathrm{Hg} & 1751 & 1673 & 5.8 & / 2 / \\ \mathrm{Cs} & 1924 & 92.5 & 0.38 & / 3 / \\ \mathrm{Rb} & 2017 & 124.5 & 0.29 & / 3 / \\ \mathrm{K} & 2178 & 150 & 0.17 & / 4 /\end{array}$

The critical temperatures of most metals can only be estimated and lie well above those of $\mathrm{Hg}$ and the alkali metals $\left(T_{c}>3000 \mathrm{~K}\right)$. Conventional measurements under static equilibrium conditions are impossible at such extreme values of temperature. Consequently, considerable effort has been spent to estimate the critical properties and thermophysical properties of metals. The estimation methods are based on different versions of the relation between the critical data and other thermophysical properties. The most frequently used method is the principle of corresponding states which is well established for molecular fluids, but its validity for fluid metals is open to serious questions. The theoretical assumption underlying the principle of corresponding states, that the nature of the interaction between particles is independent of the thermodynamic state of the system, is violated for metals and it is obvious from recent experiments $/ 2,3,5 /$ that there are significant differences between molecular and metallic fluids. Despite the extreme conditions at their critical points, the liquid-vapour coexistence curves of the metals $\mathrm{Hg}, \mathrm{Cs}, \mathrm{Rb}$ and $\mathrm{K}$ are now known to a resolution $\Delta \mathrm{T} / \mathrm{T}_{\mathrm{c}}=510^{-4}$. Figure 1 contrasts the coexistence curves of these metals in reduced units $\left(\rho / \rho_{c}\right.$ versus $\left.T / T_{c}\right)$ with that of the inert gases argon and xenon. The most significant feature seen is the extreme asymmetry of the coexistence curves of the alkali metals $\mathrm{K}, \mathrm{Rb}$ and $\mathrm{Cs}$ relative to that of the inert gases and $\mathrm{Hg}$. The coexistence curve of $\mathrm{Hg}$ differs from those of both the nonmetallic fluids and the alkali metals. Clearly, there is no simple law of corresponding states encompassing both molecular and metallic fluids, and furthermore reduced correlations are unlikely to hold for liquid metals as a group. However, the three alkali metals $\mathrm{K}, \mathrm{Rb}$ and $\mathrm{Cs}$ seem to have approximately their own common behaviour. 


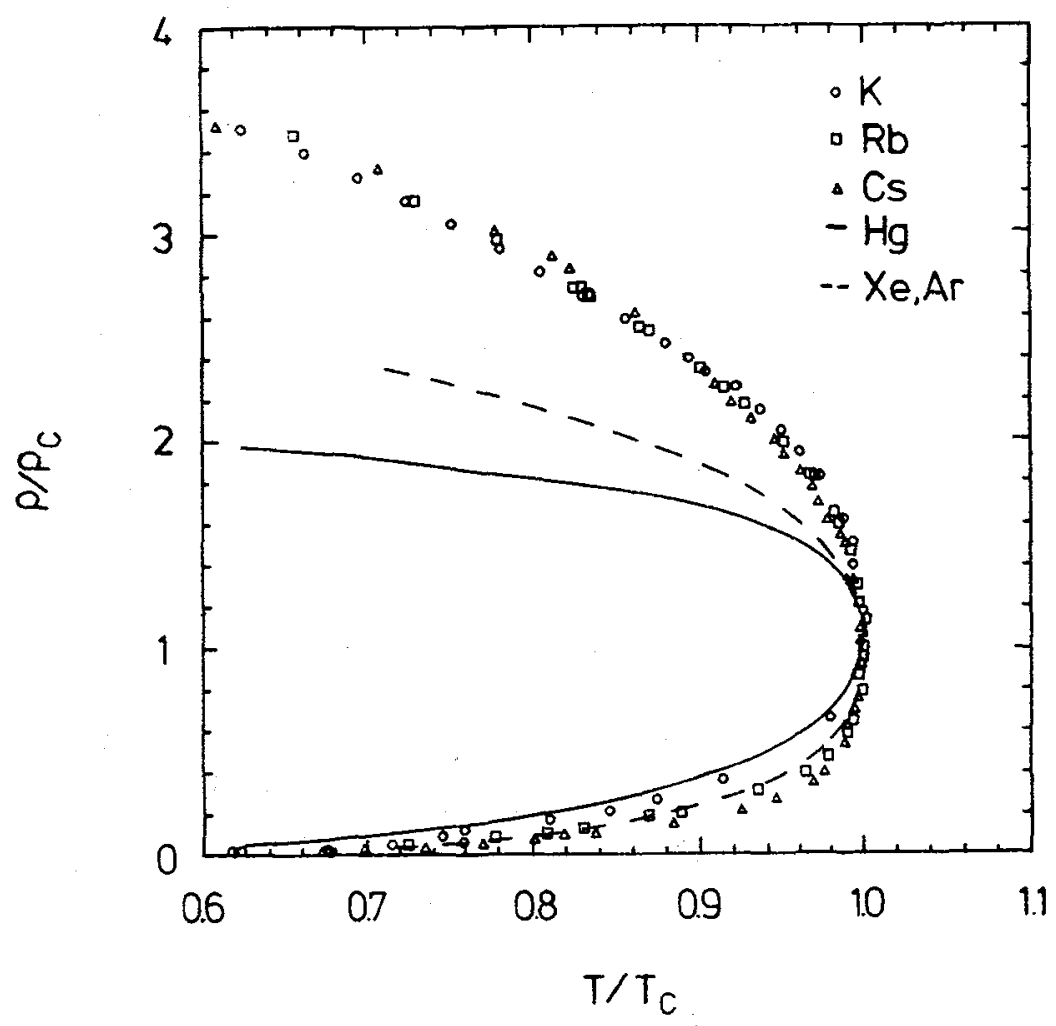

Fig. 1 The reduced densities of the coexisting vapour and liquid of the inert gases Ar and $\mathrm{Xe}$ compared with those of the metals $\mathrm{K}, \mathrm{Rb}, \mathrm{Cs}$ and $\mathrm{Hg}$.

\section{The Metal-Nonmetal Transition}

\section{a) Monovalent Metals}

A considerable amount of effort has been centered on the experimental and theoretical investigation of fluid alkali metals, despite the severe experimental difficulties associated with their highly reactive nature. One reason for their importance is the monovalency of alkali metals. With a single electron per atom they closely resemble the expanded crystals with half full bands considered by Mott $/ 6 /$ in his original discussion of the metal-nonmetal transition due to correlation. Most of the effort has focussed on cesium because of its relatively low critical temperature.

The physical consequences of the strongly state-dependent electronic structure in course of the metal-insulator transition are vividly illustrated by data such as those displayed in Figure 2 . These plots show a selection of the most accurate number density $\mathrm{n}$ (Figure 2a) and DC electrical conductivity $\sigma$ (Figure $2 b$ ) data in form of isotherms as a function of pressure at sub- and supercritical temperatures. Near the critical point the conductivity drops sharply showing a strong effect of the critical point phase transition on the electronic structure. Nevertheless, there is no indication of a discontinuity in the electronic character within the present limits of experimental resolution. Except across the liquid-vapour phase boundary, the metal-insulator transition is 
(a)

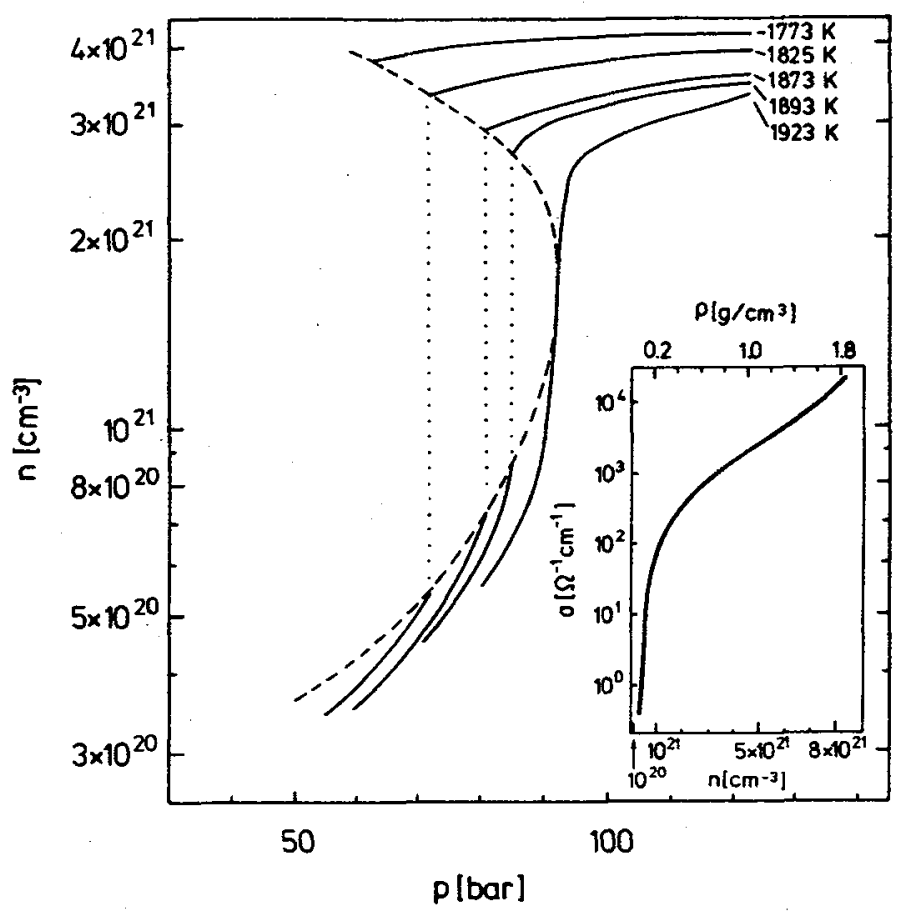

(b)

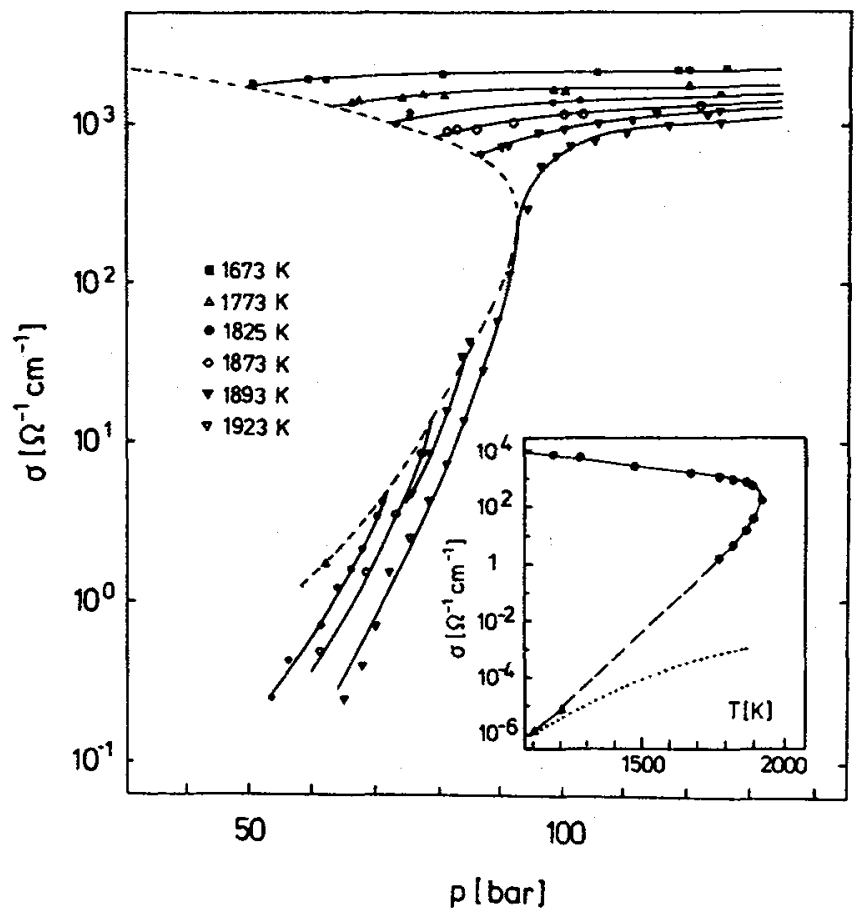

Fig. 2 (a) Equation-of-state data of fluid cesium at subcritical and supercritical temperatures as a function of pressure (inset: $\sigma$ as a function of density). (b) Electrical conductivity $\sigma$ isotherms of fluid cesium (inset: ... Saha equation, own data, $\Lambda / 28 /$ ). 
continuous. The data shown in Figure 2 illustrate the qualitative relationship between rapid variations in the conductivity $\sigma$ and the density $\rho$ and suggest that density is the dominant factor governing the metal-insulator transition.

To help distinguish the effects of density and temperature changes, it is useful to consider the schematic plot of conductivity isotherms versus density given in the inset of Figure 2a. These data show rather striking density dependences of the isotherms whereas the temperature dependence becomes invisibly small in such a plot. At the highest density $n=8.310^{21} \mathrm{~cm}^{-3}$, corresponding to the liquid range near room temperature, the conductivity $\sigma$ is about $10^{4} \Omega^{-1} \mathrm{~cm}^{-1}$ and free electron theory gives an electron mean free path $\mathrm{L}$ which exceeds the mean interatomic spacing a. Use of the measured values of the liquid structure factor $/ 7 /$ together with a model pseudopotential form factor /7/ permits the calculation of $\sigma$ within the framework of the nearlyfree-electron (NFE) model. Comparison between the calculated and the experimental $\sigma$-data shows that the NFE-theory provides a good account of the variation of $\sigma$ or, strictly speaking, relates $\sigma$ and the structure factor for densities larger than $\mathrm{n} \geq 6.010^{21} \mathrm{~cm}^{-3}$. At lower densities, the measured conductivity is clearly lower than the value calculated employing the NFE-model. The gradual failure of the NFE-model at low densities is probably not due to the breakdown of the NFE-condition $L>$ a at $6.010^{21} \mathrm{~cm}^{-3}$. Estimates of $\mathrm{L}$ from the conductivity indicate that this condition is only reached in the density range $n=3.610^{21} \mathrm{~cm}^{-3}$. Rather, the NFE-breakdown is more likely to reflect the increased importance of electron correlation below this density.

Magnetic susceptibility- /8/, NMR- /9/ and optical reflectivity /10/ studies of low-density liquid cesium have yielded evidence of strong electron-electron-correlations in the form of susceptibility enhancements and antiferromagnetic spin fluctuations. The susceptibility, NMR and optical data show a relatively sharp onset of a correlation enhancement of the effective mass at a density $\mathbf{n}=$ $6.010^{21} \mathrm{~cm}^{-3}$. The combined analysis of the magnetic and NMR data implies a correlation enhancement of the effective mass $m_{e f f}=5 m_{0}$, for example, at a density of $3.610^{21} \mathrm{~cm}^{-3}$ (the density at the liquid-vapour critical point is $n_{c}=1.7210^{21} \mathrm{~cm}^{-3}$ ) $/ 11 /$. For still smaller densities (i.e. smaller than $3.610^{21} \mathrm{~cm}^{-3}$ ) the magnetic susceptibility measurements $/ 8 /$ and quantum statistical calculations /12/ indicate the presence of localized species, such as Cs-atoms and $\mathrm{Cs}_{2}$ dimers, the concentration of which is increasing near the critical point, thus leading to large deviations of the electrical conductivity from NFE-behaviour.

The magnetic susceptibility and optical absorption measurements for alkali metals in the vapour phase show that a high concentration of molecular associates forms as the vapour density increases. These clusters have ionization energies substantially lower and electron affinities substantially higher than the corresponding values of the single atom. This effectively increases the probability of electron transfer from an atom to a large cluster and can account for the relatively large conductivity values of the coexisting cesium vapour phase close to the critical point. The inset in Fig. $2 \mathrm{~b}$ shows $\sigma$-data of the coexisting liquid and vapour phases up to the critical point in comparison with the conductivities calculated for the thermal equilibrium ionization fraction of monoatomic cesium with the vacuum ionization potential of $3.89 \mathrm{eV}$ (Sahaequation). The large ionization fraction close to the critical point is consistent with the assumption that the formation of charged clusters is essential for the interpretation of the conductivity data of the vapour. 


\section{b) Divalent Metals}

As Table 1 shows, mercury has the lowest critical temperature of any fluid metal. For this reason it has proven to be an important substance for investigation of the metal-insulator transition of a divalent metal at low density and for the study of the relationship between microscopic electronic structure and the liquid-vapour phase transition.

Density and conductivity isotherms of mercury as a function of pressure bear a qualitative similarity to those of cesium shown in Figures $2 \mathrm{a}$ and $2 \mathrm{~b}$. An important quantitative difference, however, is the value of the conductivity at the critical point. The value for mercury is roughly two orders of magnitude lower than that for cesium. This observation demonstrates that there is no universal behaviour of the electronic properties of fluid metals.

The separate effects of temperature and density are shown in Figure 3 representing isothermal plots of $\sigma$ versus $\rho$. At the highest density $\rho=13.6 \mathrm{gcm}^{-3}$, corresponding to the liquid range near room temperature, the conductivity is about $10^{4} \Omega^{-1} \mathrm{~cm}^{-1}$ and free electron theory gives an electron mean free path $L$ of $7 \AA$ which exceeds only slightly the mean interatomic spacing a. Application of the NFE-model leads again to the conclusion that $\sigma$ can be satisfactorily explained within this theory. Mercury is thus essentially a NFE metal, despite the comparatively small mean free path. The NFE character is further confirmed by the observation that in this density range,

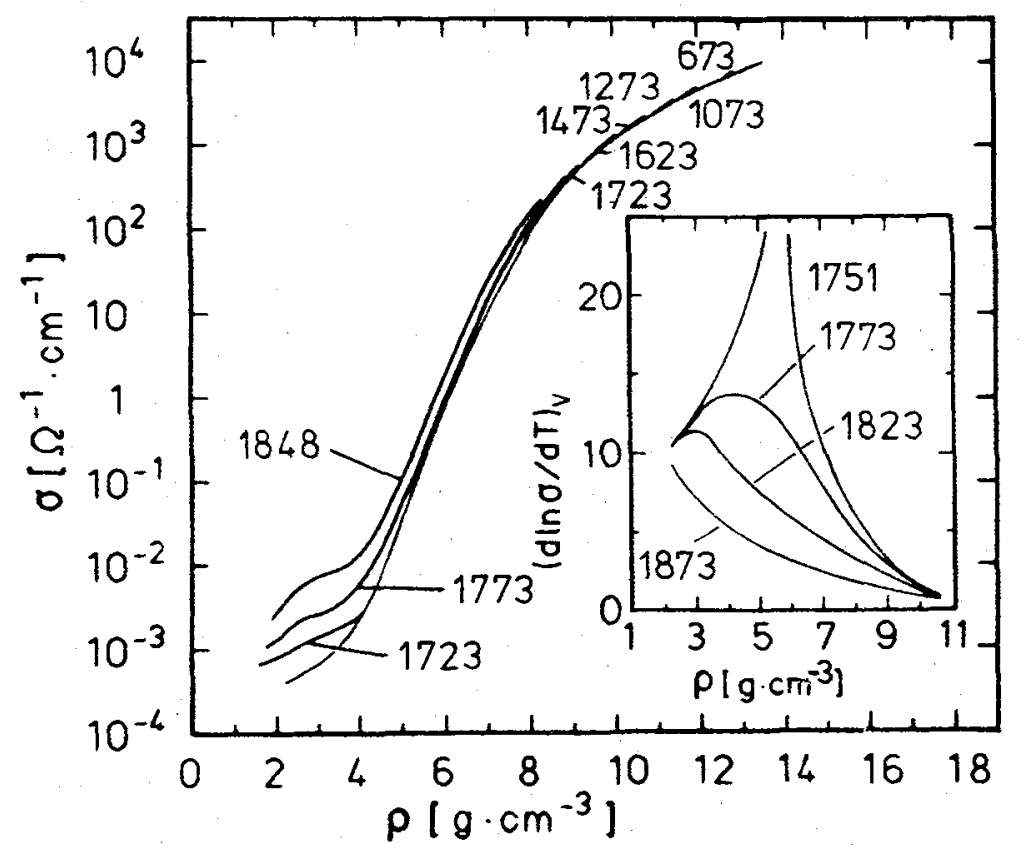

Fig. 3 Electrical conductivity $\sigma$ of fluid mercury at constant sub- and supercritical temperatures as a function of density $\rho$. The inset shows the constant volume temperature coefficient $(\partial \ln \sigma / \partial \mathrm{T})_{\mathrm{V}}$ at constant temperatures as a function of density. 
the low frequency optical conductivity $\sigma(\omega)$ shows Drude-like behaviour /13/ and the Hall coefficient $R_{H} / 14 /$ retains the free electron value. A comparison of the density dependence of $\sigma$, $\sigma(\omega), \mathrm{R}_{\mathrm{H}}$, and the Knight shift $\mathrm{K} / 15$ / shows that for densities down to about $11 \mathrm{gcm}^{-3}$ the properties of mercury can be described by the NFE-theory of metals but, with further decreasing density, a rather gradual diminution of metallic properties occurs in the density range between 11 and $9 \mathrm{gcm}^{-3}$. For still smaller densities, the behaviour or $\sigma, \sigma(\omega)$, and $\mathrm{K}$ is characteristic of a substance with semiconducting properties.

As is well known, this type of metal-semiconductor transition is predicted by the Bloch-Wilson band model to occur for an expanded divalent metal such as mercury when the 6s-valence- and 6p-conduction bands no longer overlap. In a crystal, a real energy gap appears and widens as the density decreases. It is generally assumed that the main features of the crystalline model survive in the liquid state with the band edges smeared out by disorder. Thus, the density of states $\mathrm{N}(\mathrm{E})$ is expected to tail into the gap owing to the loss of long range order. The tails overlap in the region of the Fermi energy $E_{F}$ replacing the real energy gap of the crystal by a pseudogap or a minimum in $\mathrm{N}(\mathrm{E})$ at $\mathrm{E}_{\mathrm{F}}$. The pseudogap depends strongly on density. When the magnitude of $\mathrm{N}(\mathrm{E})$ in the pseudogap decreases with sufficient expansion to a negligibly small value, the optical properties of mercury must become compatible with the opening-up of an energy gap. The latter is observed for expanded mercury for densities lower than $9 \mathrm{~g} \mathrm{~cm}^{-3}$. For these densities the shape of the $\sigma(\omega)$-curves is characteristic of a substance with either a real energy gap or with a range of energy, which is so thinly populated with states that their contribution to the optical properties is negligibly small.

In principle, one might hope to determine the energy gap experimentally by identifying it with the activation energy of the conductivity which successfully describes the temperature dependence of the conductivity in crystalline and amorphous solid semiconductors. But in high temperature liquids like mercury not too far from its critical point, strong fluctuations in local density become important. Hence, the application of solid state concepts suffers from serious limitations. This is immediately evident on consideration of the constant-volume temperature coefficient $(\partial \ln \sigma / \partial \mathrm{T})_{\mathrm{V}}$ plotted versus the density in the inset of Figure 3 for various temperatures. The strong temperature dependence of $(\partial \ln \sigma / \partial \mathrm{T})_{\mathrm{V}}$ around the critical density of $5.8 \mathrm{gcm}^{-3}$ is an obvious indication of the strong interplay between the liquid-vapour density fluctuations and the electronic characteristics.

The dominance of density fluctuations in determining the physical properties of metals in the critical region becomes especially evident in the behaviour of the optical properties of mercury /16/. At very low densities a line-spectrum is observed with the main absorption lines at $4.89 \mathrm{eV}$ and $6.7 \mathrm{eV}$ corresponding to transitions between the $6 \mathrm{~s}$ ground state and the triplet and singlet state of the $\mathrm{Hg}$ atom. As the density is increased, the sharp lines broaden due to interactions with neighbouring atoms, resulting in a relatively steep absorption edge, which moves rapidly to lower energies with increasing density. Detailed analysis of the data $/ 17,18 /$ shows, in fact, that a uniform density increase is insufficient to explain the observed line broadening. Clusters of atoms created by density fluctuations have to be explicitly taken into account. The absorption edge is thus explained in terms of absorption by excitonic states of large randomly distributed clusters. 
From the large values of the absorption coefficient it can be concluded that the singlet exciton $\left(6^{1} \mathbf{P}_{1}\right)$ with large oscillator strength broadens faster than the triplet exciton $\left(6^{3} \mathbf{P}_{1}\right)$ with small oscillator strength.

The shift of the effective optical gap and temperature- and density-induced changes in the shape of the optical absorption spectrum can be viewed equivalently in terms of a linear enhancement of $\epsilon_{1}$. Specifically, $\epsilon_{1}$ may be obtained from the standard Kramers-Kronig integral over the frequency-dependent optical conductivity. Experimental data for $\epsilon_{1}$ of mercury, at constant photon energy of $1.27 \mathrm{eV}$ are shown in Figure 4 in the form of isochores plotted versus temperature. There is an obvious indication in these data of the interplay between the critical density fluctuations and the behaviour of $\epsilon_{1}$. At the lowest metallic densities $\epsilon_{1}$ is only slightly depending on temperature and follows the Clausius-Mosotti model of the dielectric constant of induced dipoles. Then there is a strongly temperature dependent upward deviation from Clausius-Mosotti behaviour. The most characteristic feature of the data is the strong temperature dependence of $\epsilon_{1}$ close to the liquid-vapour critical point region. Figure 4 shows clearly the presence of a large anomalous critical contribution to $\epsilon_{1}$ which close to the critical isochore at a temperature about $0.1 \%$ above $T_{c}$ reaches a maximum of about $70 \%$ of the background. $A$ nonmetallic substance such as $\mathrm{CO}$, in contrast, exhibits only a very weak critical dielectric anomaly of $0.1 \%$ at a corresponding distance from the critical point $/ 19 /$. This contrast reveals the strong interplay between the vapour-liquid critical point fluctuations and the large change in electronic structure associated with the gradual metal-insulator transition.

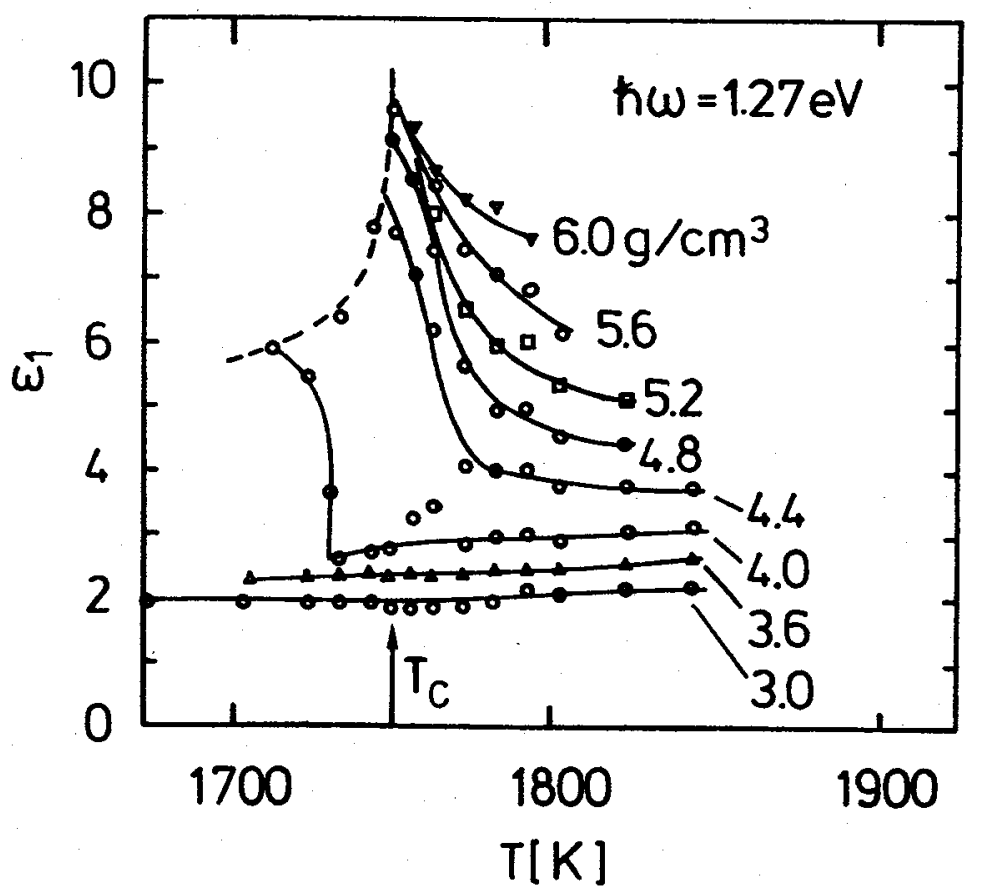

Fig. 4 The real part of the dielectric contant $\epsilon_{1}(\hbar \omega=1.27 \mathrm{eV})$ at constant densities versus temperature $\left(T_{c}=1751 \mathrm{~K}\right)$. 


\section{The Metal-Nonmetal Transition In Clusters}

The presence of large clusters in the vapour of cesium and mercury naturally raises the question whether such aggregates can be considered like macroscopic droplets having the properties of the corresponding liquid. Clusters of different sizes will be in mutual statistical equilibrium, associating and dissociating. A cluster with a small number of metal atoms differs certainly from the bulk liquid having a low density of states near the Fermi-energy and discrete energy levels which give rise to quantum size effects resembling the behaviour of molecules with only a few filled and empty levels. The highest occupied and the lowest unoccupied energy levels may be separated by energies large compared to $\mathrm{k}_{\mathrm{B}} \mathrm{T}$, so that magnetic, optical and electrical properties can be significantly different from those typical of the bulk metal. However, with increasing size of the cluster the distances between the electronic energy levels become so small compared with $\mathrm{k}_{\mathrm{B}} \mathrm{T}$ that the aggregate may be considered metallic. Thus a size-dependent gradual nonmetal to metal transition can be expected to occur in metal clusters. The critical size of a cluster for which characteristics considered as metallic are achieved depends on the detailed electronic structure of the atoms concerned.

Particularly interesting in this respect are divalent metal clusters (e.g. $\mathrm{Be}_{\mathrm{X}}, \mathrm{Mg}_{\mathrm{X}}, \mathrm{Hg}_{\mathrm{X}}$ ). These elements have a closed-shell $\mathrm{s}^{2}$ atomic configuration and have a fairly large s-p promotion energy (e.g. $\Delta_{\text {sp }}(\mathrm{Hg})=6.7 \mathrm{eV}$ ). Therefore one expects thats small clusters of mercury are insulating and bounded through weak van der Waals forces. This feature contrasts clearly with the metallic properties associated with the corresponding bulk material where metallic bonding results from the overlap between the s- and p-bands.

The relation of cluster size to the valence electronic structure of noninteracting, isolated clusters has been studied directly by employing modern supersonic beam techniques. For example, the ionization thresholds for argon-, krypton-, xenon- /20/ and mercury-clusters /21,22/ produced in supersonic beams are shown in Figure 5. Here, the ionization energies determined by energyresolved mass spectrometry and by photoelectron-photoic n- coincidence spectroscopy are plotted as a function of the cube root of the reciprocal number $\mathrm{x}$ of atoms in the cluster. This kind of plot permits a comparison of the measured size dependence of the ionization potentials $I_{p}$ with the predictions of Born's theory of solvation for the difference in solvation energies of a charge $e$ in an infinite dielectric and in a sphere of the same dielectric /23/

$$
I_{p}=W+0.5\left(e^{2} / R\right)(\epsilon-1) / \epsilon
$$

which for an ideally conducting sphere $(\epsilon \rightarrow \infty)$ leads to

$$
I_{p}=W+0.5 e^{2} / R
$$

where $\mathrm{R}$ represents the radius of an idealized sphere that contains $\mathrm{x}$ atoms, $\mathrm{W}$ represents the work function of the corresponding bulk material, e the elementary charge, and $\epsilon$ the dielectric constant of the cluster material. 
CONDENSED

STATE
ATOM

$1003020104321 \times$

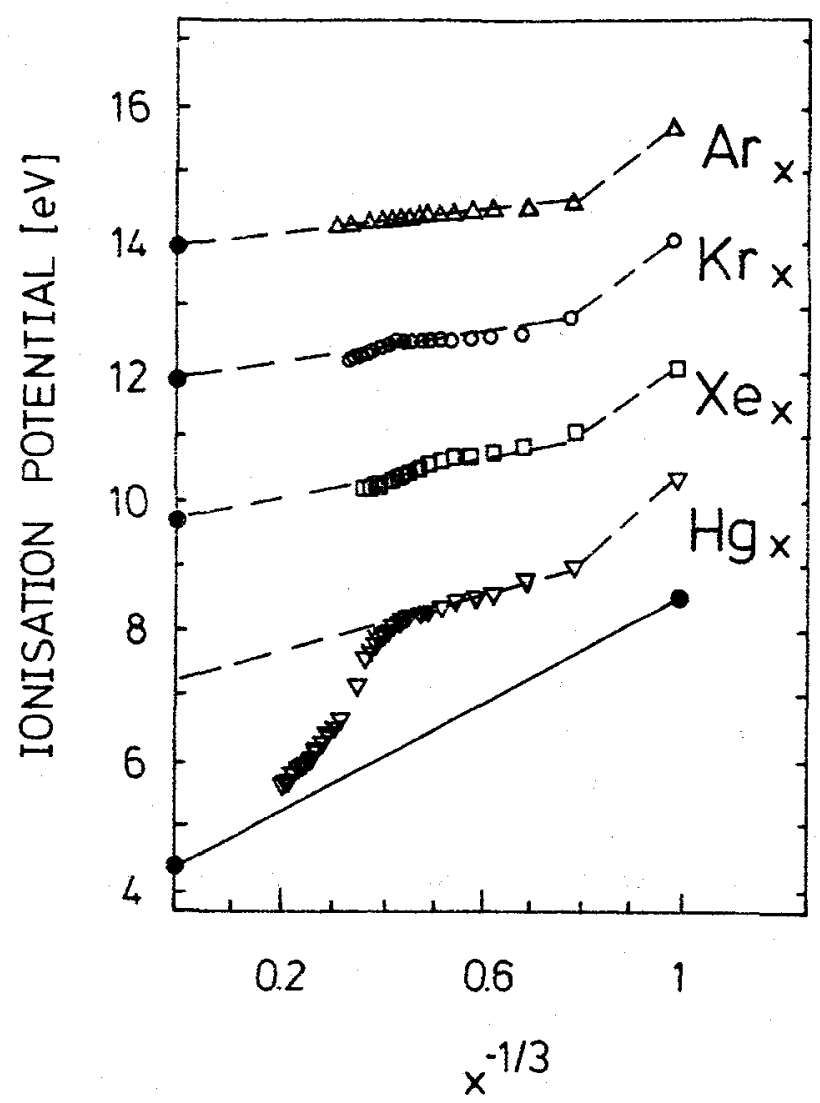

Fig. 5 Comparison of the size dependence of the effective ionization potentials of clusters of $\mathrm{Ar}, \mathrm{Kr}, \mathrm{Xe}$ and $\mathrm{Hg}$ ( $\mathrm{x}$ : number of atoms in the cluster).

It is seen at a glance at Figure 5 that in the small cluster region mercury- and the rare gas clusters show qualitatively the same behaviour (dashed curves), but the $I_{p}$-values for mercury exhibit a strong decrease by more than two $\mathrm{eV}$ in the size region between about 13 and more than 100 atoms approaching the curve (solid line) calculated employing eq. 2. The experimental results indicate that for $\mathrm{Hg}_{\mathrm{x}}$-clusters, the electronic properties of the bulk metal evolve gradually with increasing particle size in the size range $13<\mathrm{x}<110$. The strong variation in the electronic structure in course of this transformation from nonmetallic to metallic properties is also evident by studies of inner shell $5 d$-autoionization spectra $/ 24 /$, of optical absorption spectra $/ 25 /$ and of the atomic cohesion energy $/ 26 /$ of mercury clusters as a function of size. 
The strong variation in the electronic structure with cluster size influences also the kinetic features of the vapour-liquid phase transition of mercury. Measurements of the critical supersaturation for the homogeneous nucleation of mercury in the temperature range 260 to 400 $\mathrm{K} / 27$ / show that none of the current theories for homogeneous nucleation satisfactorily predicts the observed critical supersaturation. The measured values are about three orders of magnitude lower than the values predicted by the conventional Becker-Döring-Zeldovitch (BDZ) theory. It is noteworthy that the change in the value of the bulk liquid surface tension necessary to bring the classical nucleation theory in agreement with the experimental observation is about $40 \%$. In contrast, most molecular liquids require only a very small adjustment of the bulk liquid surface tension to bring nucleation theory and experiment into agreement. An important difference between mercury and the molecular fluids is that in the former the size-dependent metalnonmetal transition occurs in small clusters. Profound changes in the electronic structure of small clusters take place with increasing size, which manifest themselves in a correspondingly strong size-dependence of all properties most probable also including density and surface tension. Any rigorous theory of nucleation of metal vapours must take into account that the very existence of the size-dependent metal-nonmetal transition noticeably influences the homogenous nucleation process in supersaturated metal vapours. The kinetic formalism of the BDZ-theory can possibly be retained, but the formation Gibbs free energies of small clusters containing 2-100 atoms must be calculated ab initio employing direct statistical mechanical evaluation of the partition functions of these small clusters, a procedure which is even difficult to realize for molecular clusters as large as those important in nucleation processes. However, for metal clusters the situation is even more complicated because in contrast to most molecular clusters, for which to a first approximation the behaviour can be described by reference to a single simple dispersion interaction potential for all cluster sizes, in metal clusters the effective interaction becomes sizedependent. The occurence of the nonmetal to metal transition with increasing size implies that the nature of the interparticle interaction must change dramatically from a van der Waals-type to metallic interaction. The nucleation process in supersaturated metal vapours is fundamentally distinct from that of molecular vapours in that the interparticle potentials in the critical condensation nucleus are not quantities related to intrinsically atomic properties, but rather depend strongly on the nature of the electronic structure of the cluster.

\section{Liquid-Vapour Asymmetries in Fluid Metals}

As we have seen in the foregoing sections, the electronic structure of the two coexisting phases, liquid and vapour, of fluid metals may be fundamentally different. Indeed, at ordinary conditions far from the critical point, the vapour phase is nonmetallic and well characterized by dispersion interaction. In contrast, the coexisting liquid phase is metallic and the interaction is dominated by metallic cohesion. Both types of interactions change with the thermodynamic state, and it is this thermodynamic state-dependence of the effective interparticle interaction in course of the metalnonmetal transition which distinguishes the liquid-vapour critical point phase transition of metallic fluids from that of molecular fluids.

A number of experimental studies relevant to these issues have been reported which have shown 
that there are both important singularities, and differences between critical phenomena in molecular and metallic systems. Perhaps the most significant experiments are those on the coexistence curves of the alkali metals cesium and rubidium. As discussed previously, both fluids exhibit metallic conductivity at densities near that of the critical point, in contrast to mercury, which is more like a semiconductor or a slightly ionized plasma in the critical region.

A careful analysis of the coexistence curves shown in Figure 1 demonstrates interesting consequences of the strong thermodynamic state-dependence of an effective particle interaction in metallic fluids as the critical region is traversed. Fluid metals violate the hundred year old empirical law of rectilinear diameter over a surprisingly large temperature range. By contrast, the deviations from this law are extremely (mostly immeasurably) small for the coexisting curves of essentially all nonmetallic one-component fluids $/ 29 /$. The law states that the locus of the tie-line mid-points $\rho_{\mathrm{d}}=1 / 2\left(\rho_{\mathrm{L}}+\rho_{\mathrm{V}}\right)$ is a linear function of $\mathrm{T}$. Since both $\rho_{\mathrm{L}}$ and $\rho_{\mathrm{V}}$ approach the limiting density $\rho_{\mathrm{c}}$ at the liquid-vapour critical point, the law can be written

$$
\rho_{\mathrm{d}}-\rho_{\mathrm{c}}=\mathrm{D}_{1} \tau
$$

where $\tau=\left(T_{c^{-}} T\right) / T_{c}$ and $D_{1}$ is a constant. Modern theory of liquid-vapour critical phenomena based on renormalization group studies /30/ permits calculation of the diameter anomaly including effects of large scale density fluctuations. The theory predicts that the temperature derivative of the diameter $\mathrm{d} \rho_{\mathrm{d}} / \mathrm{dT}$, diverges at least as fast as the constant-volume specific heat $\mathrm{c}_{\mathrm{v}}$. That is, as the reduced temperature $\tau$ goes to zero, the diameter varies as

$$
\rho_{\mathrm{d}}-\rho_{\mathrm{c}}=\mathrm{D}_{0}{ }^{(1-\alpha)}+\mathrm{D}_{1} \tau+\ldots
$$

where $\alpha=0.11$ is the same exponent that describes the behaviour of the constant-volume specific heat $c_{v}$. Since $(1-\alpha)=0.89$ is not very different from unity, the true singularity is difficult to separate from the analytic temperature term. The coefficient $D_{1}$ does not even have to be much larger than $D_{0}$ for the analytic term to dominate the entire range accessible to experimentation. The latter causes the difficulty in observing the (1- $\alpha$ )-singularity for most nonmetallic fluids (see e. g. the inert gases $\mathrm{Ar}$ and $\mathrm{Xe}$ in Figure 1). And it was only with high-precision experiments on $\mathrm{Ne}, \mathrm{N}_{2}, \mathrm{C}_{2} \mathrm{H}_{4}$ and $\mathrm{C}_{2} \mathrm{H}_{6} / 29 /$ that it was possible to reveal that singularities with the predicted exponent $(1-\alpha)$ do indeed exist for nonconducting $/ 31 /$ molecular fluids, i.e. that $D_{0} \neq 0$ for these fluids as well. Analysis of these data /31/ has led to the suggestion that many-body interactions lead to the anomalous $\tau^{(1-\alpha)}$-term in these fluids. In particular, it is believed that the symmetrybreaking present in these fluids due to many body dispersion forces may be understood in terms of a thermodynamic state dependent effective pair interaction. Consequently, there seems to be a natural connection between this explanation and the observation of very large amplitudes of the diameter anomalies in fluid metals where the occurrence of the metal-nonmetal transition implies a strong variation of the interparticle interaction.

It is evident from a glance at Figure 6 that the diameter anomalies in the alkali metals, cesium and rubidium, are characterized by exponents $(1-\alpha)$. The anomalies are so strong that a nearly 


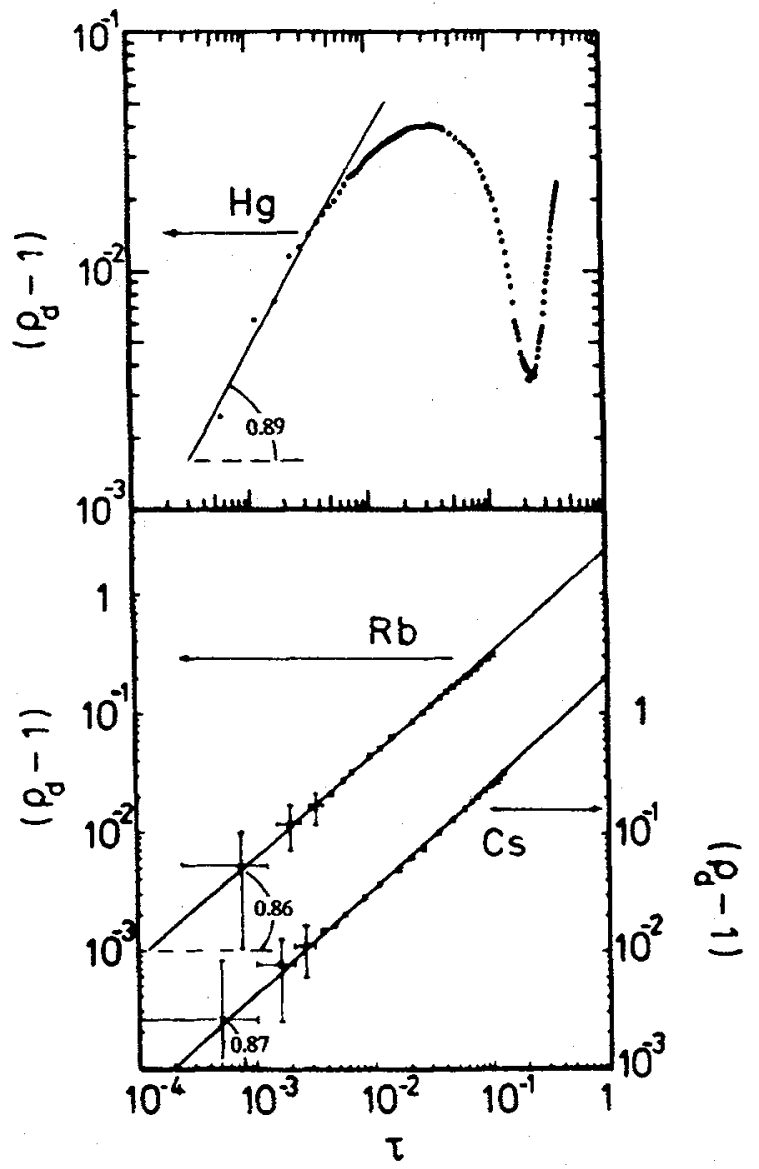

Fig. 6 Power-law analysis of the diameter of $\mathrm{Hg}, \mathrm{Rb}$ and $\mathrm{Cs}$.

pure power-law behaviour is seen over several decades in the reduced temperature $\tau$. It is certainly tempting to speculate $/ 32 /$ that the strong dependence on density of the effective interparticle potentials in the range of the gradual metal-insulator transition is responsible for the large amplitude $D_{0}$ of the $r^{(1-\alpha)}$-term in cesium and rubidium.

The liquid-vapour coexistence curve of mercury is known with an accuracy comparable to that of the alkali metals. It is evident from Figure 1 that the coexistence curve of mercury is much more symmetric than that of cesium and rubidium. Far below the critical temperature, the diameter has a positive slope as is observed for molecular fluids and for the alkali metals. In this region of temperature, when the density difference between the coexisting phases is large, the coexisting liquid is metallic $\left(\rho_{\mathrm{L}} \geq 11 \mathrm{gcm}^{-3}\right)$, while the insulating vapour consists of atoms interacting through weak van der Waals forces. At higher temperatures, where the liquid is in the electronic transition range, the diameter actually slopes towards higher densities, opposite to the behaviour of molecular fluids and fluid cesium and rubidium. 
It is seen from Figure 6 that close to the critical point of mercury the behaviour of the diameter is consistent with the $\mathrm{D}_{0} \tau^{(1-\alpha)}$-term with a positive $\mathrm{D}_{0}$. The competing variations of the electronic structure of mercury with density and temperature cause a strong wiggle at intermediate values of the reduced temperature $\tau$. It seems reasonable to assume that the skewing of the diameter towards higher densities in the metal-nonmetal density transition region between 11 and $8 \mathrm{gcm}^{-3}$ is connected with the strong volume dependence of the energy gap between the 6s- and 6p-bands which leads to a contribution to the configurational or thermal pressure of the system. The broadening of the $6 \mathrm{~s}$-band with decreasing volume is a result of the repulsive forces between mercury atoms at small interatomic separations. Consequently, excitation of electrons into the 6 p-state, whose energy is decreasing with volume, has the effect of removing some of the repulsion, thereby lowering the pressure. The decrease in repulsion may be viewed as a decrease in the effective hard core diameter of the atoms or as a softening in the effective interparticle forces.

The presence of the strong liquid-vapour interparticle asymmetry in metals does not, however, imply that the shapes of their coexistence curves cannot be described with the same exponents $\beta$ as molecular fluids in equation (5)

$$
\rho_{\mathrm{L}}-\rho_{\mathrm{V}}=\mathrm{B} \tau^{\beta}
$$

which describes the divergence of the order parameter approaching the critical point. Any speculation that the critical points of metals could fall into a different universality class than the insulating molecular fluids is clearly not true. The experimentally determined exponents $\beta$ for Cs, $\mathrm{Rb}$ and $\mathrm{Hg}$ lie between $0.35-0.36 / 5 /$, a value only slightly higher than that found for the threedimensional Ising-model. The presence of strong state-dependent interactions in metals becomes visible mainly in the behaviour of the diameter. The main difference between the coexisting curves of molecular and metallic fluids is the magnitude of the coefficient $D_{0}$ in equation (4).

\section{References}

/1/ MARCH, N, H, "Liquid Metals", Cambridge Monographs on Math. Phys., Cambridge University Press, Cambridge 1990

/2/ GÖTZLAFF, W, SCHÖNHERR, G, and HENSEL, F, Z. Phys. Chem. N. F. 156 (1988) 219

/3/ JÜNGST, S, KNUTH, B, and HENSEL, F, Phys. Rev. Lett. 55 (1985) 2160

/4/ HOHL, G, F, Doctoral Thesis, University of Marburg, Marburg 1992

/5/ HENSEL, F, J.Phys.: Condens. Matter 2 (1990) 33

/6/ MOTT, N, F, "Metal-Insulator Transitions", Taylor and Francis, London, 1974

/7/ WINTER, R, BODENSTEINER, T, GLÄSER, W, and HENSEL, F, Ber. Bunsenges. Phys. Chem. 91 (1987) 1327

/8/ FREYLAND, W, Phys.Rev. 20 (1979) 5104 
/9/ WARREN, W, W, JR., BRENNERT, G, F, and EL-HANANY, U, Phys. Rev. B 39 (1989) 4038

/10/ KNUTH, B, and HENSEL, F, High Pressure Research 5 (1990) 552

/11/ WARREN, W, W, KNUTH, B, and HENSEL, F, APS-March Meeting, March 1991, to be published

/12/ REDMER, R, and RÖPKE, G, Contrib. Plasma Phys. 29 (1989) 343

/13/ HEFNER, W, SCHMUTZLER, R, W, and HENSEL, F, J. Phys. Paris Colloq. C8 41 (1980) 62

/14/ EVAN, U, and JORTNER, J, Phys. Rev. Lett. 28 (1972) 31

/15/ WARREN, W, W, and HENSEL, F, Phys. Rev. B 26 (1982) 5980

/16/ UCHTMANN, H, BRUSIUS, U, YAO, M, and HENSEL, F, Z. Phys. Chem. N. F. $156(1988) 151$

/17/ BHATT, R, N and RICE, T, M, Phys. Rev. B 20 (1979) 466

/18/ UCHTMANN, H, POPIELAWSKI, J, and HENSEL, F, Ber. Bunsenges. Phys. Chem. 85 (1981) 555

/19/ PESTAK, M, W, and CHANG, M, H, W, Phys. Rev. Lett. 46 (1981) 939

/20/ GANTEFÖR, G, BRÖKER, G, HOLUB-KAPPE; E, and DING, A, J. Chem. Phys. 91 (1989) 7972

/21/ RADEMANN, K, KAISER, B, EVEN, U, and HENSEL, F, Phys. Rev. Lett. 59 (1987) 2319

/22/ RADEMANN, K, Ber. Bunsenges. Phys. Chem. 93 (1989) 653

/23/ MACKOR, G, NITZAU, A, and BRUS, L, E, J. Chem. Phys. 88 (1988) 5076

/24/ BRECHIGNAC, C, BROYER, M, CAHUZAC, P, DELACRETAZ,G, LABASTIE, P, WOLF, J, P, and WÖSTE, L, Chem. Phys. Lett. 120 (1985) 559; ibid 60 (1988) 275

/25/ SCHLAUF, M, Doctoral Thesis, University of Marburg, 1989

/26/ HABERLAND, H, KORNMEIER, H, LANGOSCH, H, OSCHWALD, $\mathrm{M}$ and TANNER, G, J. Chem. Soc. Faraday Trans., in press

/27/ MARTENS, J, UCHTMANN, H and HENSEL, F, J. Phys. Chem. 91 (1987) 2489

/28/ BORZHIEVSKII, A, A, SECHENOV, V, A, and KHORUNZHENKO, V, I,

Teplofizika Vysokikh Temperatur 26 (1988) 722

/29/ GOLDSTEIN, R, E, PAROLA, A, ASHCROFT, N, W, PESTAK, M, W, CHEN, M

H, W, DEBRUYN, J, R and BALZARIN, D, A, Phys. Rev. Lett. 58 (1987) 41

/30/ NICOLL, J, F, Phys. Rev. A 24 (1981) 2203

/31/ GOLDSTEIN, R, E, and PAROLA, A, J. Chem. Phys. 88 (1988) 7059

/32/ GOLDSTEIN, R, E, and ASHCROFT, N, W, Phys. Rev. Lett. 55 (1985) 2164 